\title{
PROMOÇÃO DA ATIVIDADE FÍSICA NUMA INSTITUIÇÃO DE ENSINO SUPERIOR
}

\author{
Promotion of physical activity in a higher education institution \\ Promoción de la actividad física en una institución de educación superior
}

\author{
Ana Quesado, ${ }^{*}$ Ana Rita Santos, ${ }^{* *}$ Inês Cecílio Soares, ${ }^{* *}$ Liliana Moreira, ${ }^{* *}$ Sandrina Pereira ${ }^{* *}$
}

\section{RESUMO}

Enquadramento: apesar da prática regular de atividade física influenciar positivamente a saúde emocional e física dos estudantes do ensino superior, a prevalência de inactividade física é elevada. Objetivos: realizar diagnóstico de situação relativamente à atividade física da comunidade académica; planear e implementar intervenções de promoção da atividade física mediante diagnóstico realizado; reduzir o sedentarismo na comunidade académica. Metodologia: investigação-ação desenvolvida numa Escola Superior de Saúde da região centro de Portugal. Amostragem não probabilística por conveniência. Este artigo descreve o 10 Ciclo desta investigação, no qual se realizou o diagnóstico de situação de prática de atividade física, com questionário de caraterização sociodemográfica, Questionário Global da Atividade Física e Focus Grupo. Elaborou-se um plano estratégico de promoção de atividade física e iniciou-se a implementação. Análise dos dados quantitativos através do Statistical Package for the Social Sciences, dos qualitativos seguiu-se os pressupostos de Bardin. Resultados: 53,52\% dos participantes eram ativos insuficientes, $24,65 \%$ inactivos; com média de tempo sentados 6,86 horas. No Focus Grupo sugeriram implementação de atividades em grupo e do "Gymbreak". Conclusão: a conceção do plano assentou nos eixos de intervenção integrados na estratégia nacional para actividade física, respondendo às recomendações da Direção-Geral da Saúde e envolvendo ativamente a comunidade académica.

Palavras-chave: exercício físico; promoção da saúde; instituições de ensino superior

*MsC, Professora Adjunta na Escola Superior de Saúde Norte da Cruz Vermelha Portuguesa, Doutoranda em Enfermagem na Universidade Católica Portuguesa. https://orcid.org/0000-0003-2234-4720 autor de correspondência: ana.quesado@essnortecvp.pt

** RN, Enfermeira, Escola Superior de Saúde Norte da Cruz Vermelha Portuguesa
Como referenciar:

Quesado, A., Santos, A.R., Soares, I.C., Moreira, L., \& Pereira, S. (2020). Promoção da atividade física numa instituição de ensino superior. Revista de Investigação \& Inovação em Saúde, vol.3(2). 49-60. doi.org/10.37914/riis.v3i2.91

\section{ABSTRACT}

Background: although regular physical activity positively influences the emotional and physical health of higher education students, the prevalence of physical inactivity is high. Objectives: perform a diagnosis of the situation regarding the physical activity of the academic community; plan and implement interventions to promote physical activity through diagnosis; reduce sedentary lifestyle in the academic community. Methodology: action research developed at a School of Health in the central region of Portugal. Non-probabilistic sampling for convenience. This article describes the 1st Cycle of this investigation, in which the diagnosis of physical activity was carried out, using a socio-demographic questionnaire, Global Physical Activity Questionnaire and Focus Group. A strategic plan for promoting physical activity was developed and implementation started. Analysis of quantitative data through the Statistical Package for the Social Sciences, for qualitative data, Bardin's assumptions were followed. Results: $53.52 \%$ of the participants were insufficiently active, $24.65 \%$ were inactive; with an average sitting time of 6.86 hours. In Focus Group they suggested implementing group activities and the "Gymbreak". Conclusion: the design of the plan was based on the intervention axes integrated in the national strategy for physical activity, responding to the recommendations of the Directorate-General for Health and actively involving the academic community.

Keywords: exercise; health promotion; higher education institutions

\section{RESUMEN}

Marco contextual: aunque la actividad física regular influye positivamente en la salud física y emocional de los estudiantes de educación superior, la prevalencia de la inactividad física es alta. Objetivos: realizar un diagnóstico de la situación de la actividad física de la comunidad académica; planificar e implementar intervenciones para promover la actividad física a través del diagnóstico; reducir el sedentarismo en la comunidad académica. Metodología: investigación para la Acción desarrollado en una Escuela de Salud en la región central de Portugal. Muestreo no probabilístico por conveniencia. Este artículo describe el primer ciclo de esta investigación, en el que se realizó el diagnóstico de actividad física, mediante un cuestionario sociodemográfico, Cuestionario Global de Actividad Física y Focus Group. Se desarrolló un plan estratégico para promover la actividad física y se inició la implementación. Análisis de datos cuantitativos a través del Statistical Package for the Social Sciences, para datos cualitativos, se siguieron los supuestos de Bardin. Resultados: el 53,52\% de los participantes no estaban suficientemente activos, el 24,65\% estaban inactivos; con un tiempo medio sentado de 6,86 horas. En Focus Group sugirieron implementar actividades grupales y el "Gymbreak". Conclusión: el diseño del plan se basó en los ejes de intervención integrados en la estrategia nacional de actividad física, respondiendo a las recomendaciones de la Dirección General de Salud e involucrando activamente a la comunidad académica.

Palabras clave: ejercicio físico; promoción de la salud; instituciones de enseñanza superior 


\section{Promoção da atividade física numa instituição de ensino superior}

\section{INTRODUÇÃO}

A atividade física da sociedade moderna decresce a passos largos, tornando-se num grave problema de saúde pública. Sabe-se que $70 \%$ da população mundial é sedentária, estimando-se que dois milhões de mortes por ano sejam causadas pela não adesão à prática de atividade física (Organização Mundial de Saúde [OMS], 2018; Direcção-Geral da Saúde, 2017).

A atividade física configura-se como componente relevante na prevenção de doenças crónicas não transmissíveis, como a diabetes mellitus tipo 2, doenças cardiovasculares, doenças respiratórias crónicas e cancro, atuando ainda como fator de proteção do excesso de peso e da obesidade (DirecçãoGeral da Saúde, 2017; OMS, 2015; Pires, Mussi, Cerqueira, Pitanga, \& Silva, 2013).

Sendo a meta recomendada pela OMS para adultos saudáveis com idade entre os 18 e os 65 anos de 30 minutos de atividade física de intensidade moderada 5 dias por semana, ou pelo menos 20 minutos de atividade física de intensidade vigorosa 3 dias por semana. A quantidade necessária de atividade física pode ser acumulada em sessões de pelo menos 10 minutos e poderá compreender uma combinação de períodos de intensidades moderada e vigorosa. Deverão ainda ser acrescentadas atividades que permitam melhorar a força muscular e a resistência, 2 a 3 dias por semana. Para adultos com mais de 65 anos, deverão em princípio ser alcançadas metas idênticas às de adultos mais jovens (Direcção-Geral da Saúde, 2017; Instituto Desporto de Portugal, 2009).

É durante as duas primeiras décadas de vida que se adquire e consolidam os hábitos de vida que irão perdurar até à terceira idade. No entanto, durante esse período os jovens apresentam comportamento sedentário, existindo a tendência desses hábitos aumentarem a cada década vivida, contribuindo para o aparecimento de doenças em idades precoces (Nunes, 2015; Tavares, 2014).

Neste âmbito, os estudantes do Ensino Superior são um grupo que requer uma atenção especial, visto existir evidência que a partir dos 17 ou 18 anos reduzem a adesão à participação em atividades físicas, relacionado com o início da vida universitária, o aumento da exigência e envolvimento ao nível educativo, a possível deslocação de local de residência habitual que promovem o afastamento da prática de atividade física, com impacto na suspensão dos benefícios da atividade física na sua saúde (Giesta \& Fonte, 2016).

Estudos realizados com estudantes do curso de licenciatura em enfermagem apontam para a necessidade de orientações e estímulos para a prática de atividade física ao longo do percurso académico, visando a sua valorização na vida quotidiana dos estudantes. A alta prevalência do sedentarismo nos finalistas desse curso sugeriu que o processo de formação em enfermagem não logrou bons resultados na modificação do estilo de vida dos estudantes (Nunes, 2015). Dos resultados destes estudos emergiu a reflexão sobre a importância do desenvolvimento de competências, durante o processo de formação académica, de forma a que os futuros enfermeiros incentivem a adoção de comportamentos saudáveis às pessoas que serão alvo dos seus cuidados profissionais (Nunes, 2015; Tavares, 2014).

Atualmente, a saúde e o bem-estar das comunidades académicas já são prioridades estratégicas de muitas instituições de Ensino Superior, que têm aceite o desafio do movimento das Universidades e Escolas 
Promotoras de Saúde (UEPS) da OMS, inspirados na Carta de Ottawa para a Promoção da Saúde, o qual tem alcançado um crescente interesse a nível nacional e internacional (Soares, Pereira \& Canavarro 2016).

Neste contexto, a Escola Superior de Saúde em questão não é exceção, evidenciando preocupação quanto ao estilo de vida e saúde da sua comunidade académica, que se reflete na missão desta instituição, a qual vai além do desenvolvimento do ensino, investigação, a aprendizagem ao longo da vida, a prestação de serviços à comunidade e inclui a promoção de políticas de saúde e bem-estar que contribuam para um contexto académico salutogénico. O seu projeto educativo, alinhado com a missão, orienta-se para a formação cultural, artística e desportiva, entre outros, tendo como referência a promoção de políticas de saúde e bem-estar que contribuam para um contexto académico salutogénico (Príncipe \& Ferreira, 2015). Neste enquadramento, o “Plano de Desenvolvimento Estratégico 2015/2019" desta instituição integrou nos seus objetivos 0 aumento da atividade física dos estudantes, enunciando: "Intensificar uma política da prática desportiva, cultural e recreativa a um número cada vez maior de estudantes" (Príncipe \& Ferreira, 2015, p. 19). Este artigo tem como objetivos realizar diagnóstico de situação relativamente à atividade física da comunidade académica; planear e implementar intervenções de promoção da atividade física mediante diagnóstico realizado e reduzir o sedentarismo na comunidade académica.

\section{ENQUADRAMENTO/FUNDAMENTAÇÃO TEÓRICA}

A entrada para o ensino superior tem grande influência no estilo de vida dos estudantes, acarretando um conjunto de transformações associadas à maior autonomia e responsabilidade na tomada de decisão Ihe são exigidas. As novas interacções sociais e adopção de novos comportamentos colocam o estudante numa posição vulnerável para atitudes de risco para a saúde (Silva, 2016). De acordo com o mesmo autor, uma das áreas que se encontra mais deficitária do estilo de vida dos estudantes do ensino superior é a actividade física.

A prática regular de atividade física deixa os estudantes, de ensino superior, mais satisfeitos com a sua vida académica, aumentando a autoestima, autoconfiança e autodisciplina, influenciando positivamente $\circ$ desenvolvimento das suas competências interpessoais, de entreajuda, saber lidar com o fracasso, contribuindo para melhorar o bemestar social e psicológico, a saúde emocional e física (Ferraz \& Pereira, 2002).

Apesar dos benefícios supracitados, estudos evidenciam que a prevalência de inatividade física entre os jovens universitários apresenta-se elevada, principalmente em estudantes do primeiro ano. Os principais aspetos associados a esse comportamento sedentário são a falta de tempo, motivação, apoio social e a distância entre os domicílios e espaços destinados a realização de exercícios. Portanto, e apesar dos estudantes frequentarem uma instituição de formação em saúde, os comportamentos preventivos podem mostram-se pouco frequentes. Especialmente com o ingresso na universidade, os estudantes relatam dispor de menos tempo para a prática de atividade física em razão do cumprimento das obrigações da vida académica (Pires, Mussi, Cerqueira, Pitanga, \& Silva, 2013). 


\section{Promoção da atividade física numa instituição de ensino superior}

As Instituições de Ensino Superior (IES) desempenham um papel fundamental enquanto agentes de promoção da saúde dos estudantes. As IES promotoras de saúde transformam a saúde e a sustentabilidade das sociedades atuais e futuras, contribuindo para o bem-estar das pessoas, ambiente e planeta (Ferreira, Mota, Brito, \& Santos, 2017).

Os profissionais de saúde devem estar preparados para prestar aconselhamento adequado sobre a atividade física relativamente às condições específicas das pessoas que atendem na sua prática clínica. Os enfermeiros têm, normalmente, uma grande proximidade com os utentes, por isso têm oportunidade de desenvolver uma interação mais produtiva. Neste sentido, torna-se fundamental integrar na formação de base e na formação contínua dos profissionais de saúde, informação sobre a necessidade da prática de atividade física, a melhor forma de a introduzir na vida quotidiana e, portanto, sobre as mudanças do estilo de vida (Instituto Desporto de Portugal, 2009).

Com base na revisão da literatura e inquietações pessoais surgem as seguintes questões de investigação: qual o diagnóstico de situação de prática de atividade física da comunidade académica, de uma instituição de ensino superior? e Quais as estratégias para promover a actividade física da comunidade académica, de uma instituição de ensino superior?

\section{METODOLOGIA}

Este estudo encontra-se a ser desenvolvido numa Escola Superior de Saúde da região centro de Portugal, sendo a população a comunidade académica no ano letivo 2019/2020. A amostragem foi não probabilística por conveniência, constituída pelos colaboradores (docentes e não docentes) e estudantes dos cursos de licenciatura em funcionamento na instituição.

Foram definidos como critérios de inclusão: ter vínculo com a instituição de colaborador ou estudante. Como critérios de exclusão definiram-se: colaboradores a tempo parcial, estudantes em regime pós-laboral e em programa de mobilidade.

De forma a responder ao objetivo proposto, optou-se como abordagem metodológica a investigação-ação. A investigação-ação (IA) caracteriza-se como um procedimento fundamental num dado contexto, pelo seu carácter colaborativo, visando lidar com um problema concreto (Cardoso, 2014).

O desenho da investigação seguiu a metodologia de IA baseada no modelo de Elliot (1991), assentando num processo cíclico dinâmico em espiral característico da IA, evidenciando o procedimento de revisão dos factos, de forma a identificar lacunas, antes de se proceder ao passo seguinte, no respetivo ciclo (Cardoso, 2014; Coutinho, Sousa, Dias, Bessa, Ferreira \& Vieira, 2009).

A implementação de cada fase de ação não foi linear, sendo fundamental, durante o desenvolvimento do processo, a revisão de todos os factos, antes de avançar para a etapa seguinte, como preconizado no modelo de Elliott (1991).

Numa primeira etapa efetivou-se o diagnóstico de situação face à atividade física da comunidade académica. Após a análise dos dados elaborou-se um Plano Estratégico de Promoção de Atividade Física para a comunidade académica da instituição, considerando os resultados do diagnóstico supracitado e a literatura relevante sobre a temática. Seguiu-se o início da implementação das estratégias de ação, percurso que se caracterizou pela estreita relação entre a reflexão e 
a metodologia de pesquisa. Com base no modelo em uso, são as hipóteses de ação que contribuem para a mudança das práticas e resolução do problema identificado (Cardoso, 2014; Coutinho, Sousa, Dias, Bessa, Ferreira \& Vieira, 2009).

No final do 1을 Ciclo, que correspondeu ao ano letivo 2019/2020, avaliaram-se as estratégias de ação, o que proporcionou a um novo esclarecimento e diagnóstico da situação problema, que será alicerce para a possível reestruturação do Plano Estratégico, entrando assim na espiral de reflexão e de ação até que os objetivos sejam alcançados, em consonância com o modelo de Elliott (1991). A sequência dos ciclos seguirá esta visão, correspondendo cada Ciclo a um ano letivo. Esta avaliação sistemática do decorrer da IA proporciona análise, reflexão, ajustes, redefinições ou modificações de acordo com as necessidades e circunstâncias do contexto, de modo a produzir ganhos mais duradouros nas mudanças e transformações previstas (Coutinho, Sousa, Dias, Bessa, Ferreira \& Vieira, 2009).

A opção metodológica utilizada caracteriza-se como um processo constantemente controlado passo a passo, durante períodos variáveis, neste sentido foi necessário recorrer a diversos instrumentos de colheita de dados, nomeadamente: questionário de caraterização sociodemográfica; versão portuguesa do Questionário Global da Atividade Física (GPAQ); Focus Grupo e notas de campo.

O questionário de caraterização sociodemográfica, elaborado para o efeito, avaliou os seguintes dados: vínculo com a instituição; idade; sexo; ano de inscrição no curso; como se desloca para a escola; tempo de deslocação entre casa/escola; peso atual e altura (IMC) com o intuito de conhecer a população estudada.
O Questionário Global de Atividade Física (GPAQ) foi elaborado e adaptado em 2002, de acordo com as definições do STEP wise approach to surveillance (STEPS), programa de padronização de métodos para colheita, análise e disseminação de dados da OMS. Este foi desenvolvido para uma ampla utilização internacional, atendendo às diferenças culturais e socioeconómicas das populações. Este instrumento foi submetido a teste de validade e reprodutividade entre 2002 a 2003 em 9 países, entre os quais Portugal (Bull, Maslin \& Armstrong, 2009).

O GPAQ abrange vários componentes da atividade física, como a intensidade, duração e frequência, e avalia três domínios nos quais a atividade física é realizada atividade física ocupacional, atividade física relacionada com o transporte/deslocações e atividade física durante o tempo recreativo ou de lazer. Permitindo ainda uma avaliação do comportamento sedentário e/ou inatividade.

Os Focus Grupo foram realizados por videoconferência, com recurso à plataforma do Microsoft Teams. No sentido de abranger uma amostra representativa da comunidade académica foram concretizados três Focus Grupo, com grupos distintos de elementos da comunidade académica, um com estudantes, outro com docentes e outro com não docentes. Para a moderação do Focus Grupo elaborouse um guião semiestruturado que integrou os seguintes temas: como criar condições para a prática de atividade física na instituição; estratégias/sugestões que permitam aumentar a atividade física da comunidade académica; questão/sugestões para promover a atividade física da comunidade no âmbito do plano de contingência vivenciado atualmente; e 


\section{Promoção da atividade física numa instituição de ensino superior}

identificação de atividades físicas de interesse dos participantes.

As alterações que surgiram no contexto, associadas à pandemia COVID-19 implicaram a reflexão sobre as ações e a realização de reajustes para a implementação, tal como caracteriza a metodologia seguida.

Os dados quantitativos colhidos foram analisados estatisticamente com recurso ao Statistical Package for the Social Sciences (SPSS) versão 2.3, os dados qualitativos foram submetidos a análise de conteúdo seguindo-se os pressupostos de Bardin (2016).

De forma a garantir os requisitos formais e éticos, foi solicitado parecer da comissão de ética da instituição, o consentimento à direção da instituição para a realização da investigação; o consentimento aos autores do questionário GPAQ e o consentimento dos participantes, sendo garantido o anonimato, a confidencialidade das informações recolhidas, assim como, os direitos fundamentais dos participantes.

\section{RESULTADOS}

Os resultados que se apresentam correspondem ao desenvolvimento do 1o Ciclo da IA. Na fase de diagnóstico decorreu entre fevereiro e junho de 2020, foram aplicados os questionários de caraterização sociodemográfica e GPAQ, aos quais responderam 178 indivíduos, o correspondente a $68 \%$ da comunidade académica em estudo.

Relativamente aos dados sociodemográficos verificouse que quanto ao vínculo $90 \%$ eram estudantes, $7 \%$ colaboradores docentes e 3\% colaboradores não docentes. A idade dos participantes variou entre os 17 e os 60 anos, destes $77 \%$ apresentavam idade inferior a 25 anos. Quanto ao género, $80 \%$ eram do sexo feminino. Relativamente ao modo de deslocação para a Escola constatou-se que 93,3\% utilizava o carro como meio de transporte; $3,3 \%$ utilizava transportes públicos e a mesma percentagem deslocava-se a pé. O tempo médio gasto no trajeto era de 23 minutos. Com base nos dados antropométricos (peso e altura) calculou-se o IMC, que de acordo com a classificação da OMS (Direção-Geral da Saúde, 2005) obteve-se os seguintes resultados: $62,9 \%$ peso normal; $16,3 \%$ apresentavam excesso de peso, verificando-se que destes $12,4 \%$ tinham pré-obesidade e 3,9\% classificavam-se como obesidade; $12,9 \%$ apresentavam baixo peso; $7,9 \%$ dos inquiridos não responderam à questão.

Em relação aos resultados do questionário GPAQ foi necessário considerar as instruções do próprio questionário, assim, na análise das questões relacionadas com a prática de atividade física, excluíram-se os questionários que correspondiam aos seguintes critérios: os questionários cuja soma diária de atividade física ultrapasse as $24 \mathrm{H}$; as respostas cujo número de dias por semana ultrapasse 7 , uma vez que a semana tem 7 dias; as respostas cujo tempo gasto a fazer atividades de intensidade vigorosa por dia ultrapasse $4 \mathrm{~h} / 240 \mathrm{~min}$./dia, tempo gasto por atletas de alta competição; as respostas cujo tempo gasto a fazer atividades de intensidade moderada por dia ultrapasse 4h/240min./dia. Após aplicação dos critérios supracitados foram excluídos 36 questionários, para a análise da prática de atividade física, validaram-se 142 questionários para essas questões.

Assim, constatou-se que $53,52 \%$ eram ativos insuficiente (praticavam atividade física, mas em quantidade inferior ao recomendado); $24,65 \%$ eram inativos (não praticavam qualquer atividade física); 
20,13\% eram ativos (praticavam atividade física equivalente ou superior aos valores recomendados pela OMS). Em relação ao comportamento sedentário, das 178 respostas consideradas válidas, verificou-se que num dia normal, os inquiridos passavam em média sentados cerca de 6,86 horas, num intervalo de variação entre 0,5 horas e 16 horas. Sendo que 78,09\% passavam 4 ou mais horas sentados durante um dia.

$\mathrm{Na}$ análise de dados colhidos durante o Focus Grupo não se verificaram diferenças significativas entre as informações colhidas nos três grupos, pelo que se apresentam os dados globais. Assim, durante os Focus Grupo todos os participantes revelaram praticar pouca atividade física e excesso de sedentarismo, justificando com a falta de tempo para o fazer.

Relativamente à questão sobre "como criar condições para a prática de atividade física na Instituição?", sugeriram propostas de criação de parcerias com entidades externas como ginásios, Câmara Municipal, Associações locais, entre outros, para cooperação em recursos humanos especializados no desporto, materiais e/ou instalações desportivas; e ainda, a criação de espaços dentro do recinto da instituição dedicados à atividade física.

Em relação à questão sobre "estratégias ou sugestões que permitam aumentar a atividade física da comunidade escolar?" foram identificadas: a promoção de atividades em grupo com a comunidade académica; envolvimento mais ativo da Associação de Académica nesta área; implementação do programa
"Gymbreak" em sala de aula; recurso a diversas formas de promoção de atividade física, nomeadamente vídeos ou pósteres e sua divulgação através dos meios de comunicação da Instituição, como página da internet, redes sociais, Microsoft Teams ou outras plataformas.

A respeito da questão sobre "sugestões para promover a atividade física da comunidade no âmbito do plano de contingência vivenciado atualmente?" as ideias manifestadas foram a implementação do programa "Gymbreak" nas aulas síncronas, via Microsoft Teams. No que concerne à "identificação de atividades físicas de interesse dos participantes" recorreu-se ao "Mentimeter", constando-se diferenças entre os três grupos. A atividade de maior interesse para os docentes foi a caminhada, seguida da natação e do voleibol. O pilates, a dança, a caminhada e a ioga foram as atividades mais referidas pelos colaboradores não docentes. No grupo dos estudantes, a caminhada foi apontada como a atividade de maior interesse seguida da dança, ginástica e bicicleta. Assim, perante análise dos três grupos constatou-se que a caminhada foi a atividade de maior interesse global, seguida da natação, pilates, zumba, voleibol e dança.

Com base na análise dos resultados obtidos na fase de diagnóstico, revisão da literatura e análise das orientações da OMS e Direção-Geral da Saúde (DGS) relativas à promoção da atividade física elaborou-se um Plano Estratégico de Promoção de Atividade Física na Instituição e que se sintetiza na tabela 1. 
Tabela 1

Síntese do Plano Estratégico de Promoção de Atividade Física na Instituição

\begin{tabular}{|c|c|}
\hline Ações & Descrição \\
\hline \multicolumn{2}{|c|}{ Eixo 1 - Promoção da Atividade Física } \\
\hline $\begin{array}{l}\text { Implementação do "Gymbreak" } \\
\text { em sala de aula. }\end{array}$ & $\begin{array}{l}\text { O “Gymbreak" corresponde a uma coreografia de exercícios de alongamento de } 3 \\
\text { minutos. Disseminação da transmissão do respetivo vídeo no final das aulas, do 1을 } \\
\text { tempo da manhã e da tarde, para ser acompanhado por docentes e estudantes. }\end{array}$ \\
\hline Lançamento de Desafios & $\begin{array}{l}\text { Implementação do "Siga o Assobio", através da divulgação do vídeo promovido } \\
\text { pela DGS, nos ecrãs disponíveis na Instituição e meios de comunicação. } \\
\text { Lançamento desafios à comunidade académica, nas redes sociais, no Microsoft } \\
\text { Teams ou presenciais, para a concretização de tarefas que envolvam a atividade } \\
\text { física. }\end{array}$ \\
\hline $\begin{array}{l}\text { Divulgação de atividades nos } \\
\text { meios de comunicação da } \\
\text { Instituição }\end{array}$ & $\begin{array}{l}\text { Divulgação nos vários meios de comunicação como página da internet, redes } \\
\text { sociais, Microsoft Teams de atividades de promoção da atividade física e } \\
\text { divulgação das realizadas. Nomeadamente o "Gymbreak". }\end{array}$ \\
\hline $\begin{array}{l}\text { Afixação de Posters } \\
\text { motivacionais e informativos }\end{array}$ & $\begin{array}{l}\text { Colocação em locais estratégicos na Instituição, pósteres motivacionais } \\
\text { promovidos pela DGS, outras entidades ou elaborados por estudantes. }\end{array}$ \\
\hline $\begin{array}{l}\text { Colocação Escadas } \\
\text { motivacionais }\end{array}$ & $\begin{array}{l}\text { Colocação de sinaléticas ao longo das escadas, com referência às calorias gastas } \\
\text { por degrau. Colocação de sinalética no elevador de incentivo ao uso das escadas. }\end{array}$ \\
\hline Criação do "Dia das Sapatilhas" & $\begin{array}{l}\text { Determinação de } 1 \text { dia por semana, para a realização conjunta de atividade física } \\
\text { diversificada, com a duração de uma hora. }\end{array}$ \\
\hline $\begin{array}{l}\text { Criação de Circuito } \\
\text { Manutenção/Gestão de espaços }\end{array}$ & $\begin{array}{l}\text { Criação de circuitos preparados para a realização de atividade física na Instituição } \\
\text { recorrendo a profissional da área. }\end{array}$ \\
\hline $\begin{array}{l}\text { Realização de Torneios } \\
\text { interescolas }\end{array}$ & Promoção e realização de torneios interescolares. \\
\hline Eixo 2 - Profissionais de Saúde & \\
\hline
\end{tabular}

\begin{tabular}{|c|c|}
\hline \multicolumn{2}{|c|}{ Eixo 2 - Profissionais de Saúde } \\
\hline Formação & $\begin{array}{l}\text { Integração no programa em unidades curriculares dos cursos as temáticas: } \\
\text { "atividade física na área da saúde", de modo a capacitar os futuros profissionais de } \\
\text { saúde no reconhecimento da relação entre a atividade física e algumas situações } \\
\text { patológicas, seus benefícios, potenciais riscos, restrições nessas situações; } \\
\text { "preparação física dos profissionais de saúde". Realização workshops nas áreas } \\
\text { supracitadas, entre outras. }\end{array}$ \\
\hline \multicolumn{2}{|c|}{ Eixo 3 - Trabalho Intersectorial } \\
\hline Criação de Parcerias & $\begin{array}{l}\text { Analise das parcerias existentes entre a Instituição, Câmara Municipal, ginásios, } \\
\text { entidades locais desportivas, culturais, recreativas e de lazer, entre outros; a fim } \\
\text { de sugerir melhorias ou propor novas parcerias no âmbito da cooperação em } \\
\text { recursos humanos especializados no desporto, materiais e/ou instalações } \\
\text { desportivas. Divulgação junto da comunidade académica. }\end{array}$ \\
\hline $\begin{array}{l}\text { Envolver a Associação de } \\
\text { Académica }\end{array}$ & $\begin{array}{l}\text { Envolvimento da Associação Académica na dinamização de atividades de } \\
\text { promoção da atividade física. }\end{array}$ \\
\hline \multicolumn{2}{|l|}{ Eixo 4 - Investigação } \\
\hline $\begin{array}{l}\text { Desenvolver Projetos de } \\
\text { investigação }\end{array}$ & $\begin{array}{l}\text { Continuidade no desenvolvimento da atual IA. Realização mais estudos de } \\
\text { investigação na Instituição, no âmbito da promoção da atividade física e saúde }\end{array}$ \\
\hline \multicolumn{2}{|l|}{ Eixo 5- Monitorização } \\
\hline $\begin{array}{l}\text { Criação de grupo de } \\
\text { monitorização }\end{array}$ & $\begin{array}{l}\text { O Plano Estratégico de Promoção de Atividade Física na Instituição, ao se } \\
\text { encontrara alicerçado na presente IA, será monitorizado através da equipa } \\
\text { investigadora e de acordo com a própria metodologia. }\end{array}$ \\
\hline
\end{tabular}

Este plano assentou nos mesmos eixos de intervenção que integram a estratégia nacional para a atividade física recomendada pela Direcção-Geral da Saúde (2017) e que correspondem: promoção da atividade física; profissionais de saúde; trabalho intersectorial; investigação e monitorização. Para cada eixo foram propostas ações. 
Após o planeamento deu-se início à implementação do passo 1 da aç̧ão com a implementação do "Gymbreak" em sala de aula. Reajustou-se e divulgouse o programa "Gymbreak" pelos docentes e estudantes através facebook e plataforma Microsoft Teams; envolveu-se a Direção de Ensino e Coordenadores dos Cursos de Licenciatura na sensibilização dos docentes para importância da sua implementação nas aulas síncronas.

Como avaliação da implementação do passo 1 da ação verificou-se que, apesar da grande divulgação do programa "Gymbreak", através dos recursos digitais da instituição e sensibilização da comunidade académica para a importância da sua implementação nas aulas síncronas, durante o período de confinamento, a adesão foi baixa.

\section{DISCUSSÃO}

Face à comunidade académica da Instituição, tendo em consideração uma amostragem significativa (68\%), constatou-se que $73,65 \%$ da comunidade académica pratica atividade física, seja de forma intencional, laboral ou recreativa ou como forma de deslocação; contudo apenas $20,13 \%$ pratica atividade física dentro dos valores recomendados pela OMS, ou seja, pratica 30 minutos por dia de atividade de intensidade moderada ou 10 minutos por dia de intensidade vigorosa. Estes dados corroboram os dados encontrados na literatura (OMS 2018; DGS 2017; Instituto Desporto de Portugal, 2009). Pela análise do comportamento sedentário constatou-se ainda que a maioria dos inquiridos está sentada cerca de 7 horas diárias em média. Portanto, contrariamente às recomendações da OMS verifica-se que esta comunidade académica é sedentária e pouco ativa o que vai ao encontro dos resultados de outros estudos neste âmbito (Nunes, 2015; Tavares, 2014).

Segundo Cardoso, Veras, Coelho \& Figueiredo (2017) todas as ações têm de ser adaptadas ao contexto concreto, refletindo as necessidades próprias dos grupos alvo e os termos em que foram segmentados. Neste contexto, relativamente às sugestões e estratégias referidas pelos diferentes participantes dos Focus Grupo, constatou-se uma sincronia entre os grupos nomeadamente, na implementação do "Gymbreak", uma vez que se trata de uma bateria de exercícios de alongamentos semelhantes a ginástica laboral, daí a sua inclusão no plano estratégico; outra sugestão consensual entre os grupos foi a determinação de um dia por semana para realização de atividade física em grupo, aberta a qualquer elemento da comunidade académica. Apesar da atividade mais consensual ter sido a caminhada, existiu grande diversidade na identificação de outras atividades entre os grupos, ficando previsto no Plano Estratégico de Promoção de Atividade Física na Instituição, atividade física variada a ser ajustada em cada momento aos participantes.

Conhecendo o contexto e a região envolvente da Instituição considerou-se viável a criação de parcerias com a Câmara Municipal, ginásios ou outras entidades locais que tenham infraestruturas e/ou profissionais da área do desporto. Corroborando Nunes (2015) quando afirma que devem ser desenvolvidos todos os esforços possíveis, no sentido de incentivar as escolas a proporcionarem atividades físicas em cooperação com parceiros da comunidade local e promover em todos os estudantes o interesse para toda a vida pela atividade física. 
Atendendo a recomendações encontradas na literatura consultada, nomeadamente o Instituto Desporto de Portugal (2009) que realça a importância da preparação de profissionais de saúde neste âmbito, - Plano integrou as sugestões de realização de workshops e integração no programa de unidades curriculares dos cursos, temáticas que abordem a atividade física na área da saúde, de modo a capacitar os futuros profissionais de saúde no reconhecimento de potenciais riscos e benefícios perante diferentes contextos patológicos, assim como, na área da preparação física dos próprios profissionais de saúde.

Com a continuidade da implementação da atual IA reconhece-se a importância de promover a atividade física nesta Instituição, com o recuso a ações diversificadas, indo ao encontro da ideia defendida por ORSIES (2018), de que as instituições de ensino superior desempenham um papel fundamental enquanto agentes de promoção da saúde dos estudantes. Advogando ainda Príncipe \& Ferreira (2015) que vão mais longe, ao referirem a importância da promoção de políticas de saúde e bem-estar que contribuam para um contexto salutogénico.

\section{CONCLUSÃO}

Concluiu-se que este plano estratégico de promoção de actividade física responde às recomendações da DGS, pelo que se organizou com base nos cinco eixos da estratégia nacional para a atividade física. No âmbito da promoção da atividade física previu-se a dinamização de atividades de forma sistemática e envolvam ativamente toda a comunidade académica. Quanto ao eixo profissionais de saúde propôs-se a integração curricular da temática nos cursos em funcionamento na instituição e em formações extracurriculares. No trabalho intersectorial a proposta foi no sentido de promover parcerias com entidades da região de influência da instituição e envolver ativamente a associação académica. Quanto à investigação o plano sugeriu a continuidade da investigação em causa, através da qual será possível monitorizar o próprio plano de forma a conhecer os ganhos em saúde associados.

Os resultados agora obtidos permitiram gerar dados para o reconhecimento da real dimensão do problema nesta comunidade académica, assim como, para a identificação de ações ajustadas às características da população-alvo, no sentido de concretizar um Plano Estratégico de Promoção de Atividade Física adaptado ao contexto.

O contexto pandémico que, entretanto, surgiu tem sido uma das grandes limitações, atendendo aos objetivos do estudo em confronto com as restrições impostas. Atendendo ao resultado da avaliação do 10 Ciclo, considera-se importante o desenvolvimento do 2o Ciclo, reforçando a sensibilização de docentes e estudantes para a efetiva implementação do "Gymbreak", avaliando a causa da baixa adesão ao mesmo; implementação do passo 2 da ação, previsto no Plano Estratégico de Promoção de Atividade Física na Instituição.

\section{REFERÊNCIAS BIBLIOGRÁFICAS}

Bardin, L. (2016). Análise de conteúdo. 3a reimp da 1ạ ed. São Paulo: Edições 70.

Bull, F. C., Maslin, T. S., \& Armstrong, T. (2009). Global physical activity questionnaire (GPAQ): nine country reliability and validity study. Journal of Physical Activity \& Health, 6(6), 790-804.

Cardoso, G. M., Veras, R. M., Coelho, M. T., \& Figueiredo, W. N. (2017). Vida universitária e atividade física: um estudo sobre a produção acadêmica. Revista 
de atenção à saúde. 15 (52), doi.10.13037/ras.vol15n52.4522

Cardoso, A.P.P.O. (2014). Inovar com a investigaçãoação: desafios para a formação de professores. Coimbra: Universidade de Coimbra. Doi: 10.14195/978-989-26-0666-8

Coutinho, C. P., Sousa, A., Dias, A., Bessa, F., Ferreira, M. J., \& Vieira, S. (2009). Investigação-ação: metodologia preferencial nas práticas educativas. Revista Psicologia, Educação e Cultura, 13 (2), 355-379. Retirado de http://repositorium.sdum.uminho.pt/bitstream/1822 /10148/1/Investiga\%c3\%a7\%c3\%a3o_Ac

\%c3\%a7\%c3\%a3o_Metodologias.PDF

Instituto Desporto de Portugal (2009). Orientações Europeias para a Atividade Física - Políticas Recomendadas para a Promoção da Saúde e do BemEstar. Lisboa: Edição do Instituto do Desporto de Portugal. Retirado de http://biblioteca.esec.pt/cdi/ebooks/docs/Orientacao \%20Europeia\%20Act\%20F\%C3\%ADsica.p df

Direção-Geral da Saúde (2017). Programa Nacional Para a Promoção Da Atividade Física. A saúde dos Portugueses 2016. Lisboa: DGS. Retirado de https://www.dgs.pt/programa-nacional-para-apromocao-da-atvidade-fisica/ficheiros-externospnpaf/rel_capitulo-pnpaf-2016-a-saude-dos-ptpdf.aspx

Elliott, J, (1991). Action Research for Educational Change, Open University Press: Milton Keynes. First published: December $1993 . \quad$ DOI: 10.1080/0141192930190510

Ferraz, M., \& Pereira, A. S. (2002). A dinâmica da personalidade e o homesickness (saudades de casa) dos jovens estudantes universitários. Psicologia, Saúde \& Doenças, 149-164. Retirado de http://www.scielo.mec.pt/pdf/psd/v3n2/v3n2a04.pdf Ferreira, F. M., Mota, L. A., Brito, I., \& Santos, M. R. (2017). Perfil de saúde dos estudantes de enfermagem: diagnóstico epidemiológico a partir do modelo PRECEDE-PROCEED. Revista de Enfermagem Referência, 15, 91-100. doi: 10.12707/RIV17047

Giesta, L., \& Fonte, C. (2016). Saúde mental e nível de atividade física em estudantes do ensino superior, Revista Psicologia Educação e Cultura, 1, 153-171. Retirado de https://bdigital.ufp.pt/bitstream/10284/7988/1/Giest a\%20\%26\%20Fonte\%20Abstract\%20201 6.pdf
Nunes, C. C. (2015). Atividade física dos estudantes de enfermagem. (Tese de Mestrado). Escola Superior de Saúde de Viseu do Politécnico de Viseu. Retirado de https://repositorio.ipv.pt/bitstream/10400.19/2840/1 /NUNES\%2C\%20CarlaCristinaGon\%C3\%A

7alves\%20DM.pdf

OMS (2015). Physical activity strategy for the WHO European Region 2016-2025. Copenhagen: WHO. Retirado de https://apps.who.int/iris/bitstream/handle/10665/32 9407/9789289051477-eng.pdf

OMS (2018). Global action plan on physical activity 2018-2030: more active people for a healthier world. Geneva: WHO. Retirado de https://apps.who.int/iris/bitstream/handle/10665/27 2722/9789241514187-eng.pdf?ua=1

ORSIES. (2018). Livro Verde sobre Responsabilidade Social. Lisboa: ORSIES. Retirado de https://orsies.forum.pt/

Pires, C. G. S., Mussi, F. C., Cerqueira, B. B., Pitanga, F. J. G., \& Silva, D. O. (2013). Prática de atividade física entre estudantes de graduação em enfermagem. Acta Paulista de Enfermagem, 26(5), 436-443. Doi: 10.1590/S0103-21002013000500006

Príncipe, F., \& Ferreira, A. (Coord.) (2015). Plano Desenvolvimento Estratégico 2015/2019. Oliveira de Azeméis: ESEnfCVPOA. Retirado de https://www.essnortecvp.pt/pt/escola/sobrenos/

Silva, A.M.M, (2016). Estilos De Vida Em Estudantes Do Ensino Superior. (Tese de Doutoramento). Instituto De Ciências Da Saúde da Universidade Católica Portuguesa. Retidado de: https://www.google.com/url?sa=t\&rct=j\&q=\&esrc=s\& source $=$ web\& $c d=\& c a d=r j a \&$ uact $=8 \&$ ved $=2$ ahUKE wiZ3 K2HuNzsAhVSoXEKHTEMCY8QFjAAegQIARAC\&url=htt ps\%3A\%2F\%2Frepositorio.ucp.pt\%2Fbitstream\%2F10 400.14\%2F24196\%2F1\%2FEstilos_de_Vida_em_Estud antes.pdf\&usg=AOvVaw2VgV2-rh5kqQbsvM8erWQI

Soares, A., Pereira, A., \& Canavarro, J. (2016) Promoção da Saúde nas Instituições de Ensino Superior Portuguesas: Reflexões e Desafios, Revista Portuguesa de Pedagogia, 2(1), 115- 137. DOI 10.14195/16478614_49-2_6

Tavares, C. F. (2014). Adaptação ao ensino superior, personalidade e otimismo em estudantes universitários do 1ㅇ ciclo de estudos. (Tese de Mestrado). Faculdade e Ciências Humanas e Sociais da Universidade Fernando Pessoa. Retirado de: 
Promoção da atividade física numa instituição de ensino superior

https://bdigital.ufp.pt/bitstream/10284/4231/1/CL\%C 3\%81UDIO\%20FILIPE\%20VALENTE\%20TAVARES\%20\%20ADAPTA\%C3\%87\%C3\%830\%20AO\%20ENSINO\%2 OSUPERIOR\%2C\%2OPERSONALIDADE\%20E\%200TIMIS MO\%20EM\%20ESTUDANTES\%20UNIVERSIT\%C3\%81RI OS\%20DO\%201\%C2\%BA\%20CICLO\%20DE\%20ESTUDO S.pdf 